\title{
Quantum phases of atomic Fermi gases with anisotropic spin-orbit coupling
}

\author{
M. Iskin ${ }^{1}$ and A. L. Subaşı ${ }^{2}$ \\ ${ }^{1}$ Department of Physics, Koç University, Rumelifeneri Yolu, 34450 Saryer, Istanbul, Turkey \\ ${ }^{2}$ Department of Physics, Faculty of Science and Letters, Istanbul Technical University, 34469 Maslak, Istanbul, Turkey
}

(Received 22 August 2011; published 13 October 2011)

\begin{abstract}
We consider a general anisotropic spin-orbit coupling and analyze the phase diagrams of both balanced and imbalanced Fermi gases for the entire BCS-BEC evolution. In the first part, we use the self-consistent mean-field theory at zero temperature, and show that the topological structure of the ground-state phase diagrams is quite robust against the effects of anisotropy. In the second part, we go beyond the mean-field description, and investigate the effects of Gaussian fluctuations near the critical temperature. This allows us to derive the time-dependent Ginzburg-Landau theory, from which we extract the effective mass of the Cooper pairs and their critical condensation temperature in the molecular BEC limit.
\end{abstract}

DOI: 10.1103/PhysRevA.84.043621

PACS number(s): 03.75.Ss, 05.30.Fk, 03.75.Hh

\section{INTRODUCTION}

The realization of BCS-BEC evolution with twocomponent atomic Fermi gases has received tremendous attention in the past decade [1,2]. In these experiments, the tuning of attractive interactions permits the ground state of the system to evolve from a weak fermion attraction BCS limit of loosely bound and largely overlapping Cooper pairs to a strong fermion attraction limit of tightly bound small bosonic molecules which undergo BEC. The main difference between the BCS-BEC evolution problem and the simple BCS theory is that the Cooper pairing is not allowed only for fermions with energies close to the Fermi energy but is also allowed for all momenta.

All of the early theoretical works were concentrated on the balanced Fermi gases, i.e., both components have the same number and mass, suggesting that the evolution is not a phase transition but a smooth crossover, and hence the name $B C S-B E C$ crossover. This prediction was then found to be in very good agreement with all of the observations [1,2]. Motivated by the experimental success with balanced Fermi gases, many of the recent theoretical works were concentrated on imbalanced (population, mass, and/or dimension) Fermi gases, suggesting various phases and phase transitions between them. Some of these predictions were also confirmed by the following experiments [3-6], opening the door for studies on more complicated systems with the hope of finding exotic phases of matter.

Arguably, one of the very promising alternate systems to investigate is the spin-orbit coupled (SOC) atomic Fermi gases $[7,8]$. This is mainly motivated by the very recent success in realizing SOC atomic BEC $[9,10]$, and by a practical proposal for generating a SOC Fermi gas with ${ }^{40} \mathrm{~K}$ atoms [11]. Some of the very recent results on these systems can be summarized as follows. For the two-body problem, it has been found that a two-body bound state exists for some types of SOC, e.g., Rashba or Dresselhaus types, even on the BCS side $\left(a_{s}<0\right)$ of a resonance [12] with arbitrarily small $a_{s} \rightarrow 0^{-}$, where $a_{s}$ is the $s$-wave scattering length. For the many-body problem, it has been found for balanced Fermi gases that the SOC increases the single-particle density of states, which in return favors the Cooper pairing so significantly that increasing the SOC, while $a_{s}$ is held fixed, eventually induces a BCS-BEC evolution even for a weakly interacting system when $a_{s} \rightarrow 0^{-}$ [13-17]. Similar to what happens in the usual BCS-BEC crossover picture of a balanced Fermi gas with increasing $1 / a_{s}$, the BCS-BEC evolution with increasing SOC turns out to be a smooth crossover but not a phase transition. Therefore, the ground state of balanced Fermi gases with or without the SOC is a topologically trivial gapped superfluid.

However, the BCS-BEC evolution with Rashba-type SOC is found to become a phase transition for population-imbalanced [18-20] and/or mass-imbalanced [21] Fermi gases. It has been found that the SOC counteracts the population imbalance, and that this competition tends to stabilize the uniform topologically nontrivial gapless superfluid phases against the phase separation. In addition, topological phase transitions associated with the appearance of momentum space regions with zero quasiparticle and quasihole energies have been found, the signatures of which could be observed in the momentum distribution or the single-particle spectral function $[19,20]$.

The anisotropic (in momentum space) nature of the SOC is also found to stabilize exotic superfluid phases. For instance, in sharp contrast to the no-SOC case where only the gapless superfluid phase supports population imbalance, both the gapless and gapped superfluid phases are found to support population imbalance in the presence of a Rashba-type SOC $[19,20]$. Similarly, again in sharp contrast to the no-SOC case where only the gapped superfluid phase supports population balance, both the gapped and gapless superfluid phases are found to support population balance in mass-imbalanced SOC Fermi gases when the mass difference becomes large enough [21].

In this paper, we extend our recent works [19,21], and study the effects of anisotropic SOC on the phase diagrams of both balanced and imbalanced Fermi gases throughout the entire BCS-BEC evolution. We analyze both zero- and finitetemperature phase diagrams, and the paper is organized as follows. First, we review the noninteracting and interacting two-body problem in Sec. II, and calculate the binding energy of the two-body bound state in vacuum. Second, we study the many-body problem in Sec. III, where we derive the meanfield theory at zero temperature, and use it to analyze the ground-state phase diagrams of imbalanced Fermi gases. Then, 
we investigate the Gaussian fluctuations in Sec. IV near the critical temperature, and calculate the effective mass of the Cooper pairs and their critical condensation temperature in the molecular BEC limit. Last, our conclusions are briefly summarized in Sec. V.

\section{TWO-BODY PROBLEM}

Before presenting our results for the many-body problem, let us first introduce the model Hamiltonian and review some of the recent results for the noninteracting and interacting two-body problem.

For the noninteracting SOC fermions, the two-body Hamiltonian (in units of $\hbar=1=k_{B}$ ) can be written as

$$
H_{0}=\sum_{\mathbf{k}} \psi_{\mathbf{k}}^{\dagger}\left(\begin{array}{cc}
\epsilon_{\mathbf{k}, \uparrow}+S_{k_{z}} & S_{\mathbf{k}_{\perp}} \\
S_{\mathbf{k}_{\perp}}^{*} & \epsilon_{\mathbf{k}, \downarrow}-S_{k_{z}}
\end{array}\right) \psi_{\mathbf{k}}
$$

where $\psi_{\mathbf{k}}^{\dagger}=\left[a_{\mathbf{k}, \uparrow}^{\dagger}, a_{\mathbf{k}, \downarrow}^{\dagger}\right]$ with $a_{\mathbf{k}, \sigma}^{\dagger}\left(a_{\mathbf{k}, \sigma}\right)$ creates (annihilates) a spin- $\sigma$ fermion with momentum $\mathbf{k}=\left(k_{x}, k_{y}, k_{z}\right), \epsilon_{\mathbf{k}, \sigma}=$ $k^{2} /(2 m)$ is the kinetic energy, and $S_{\mathbf{k}_{\perp}}=\alpha_{x} k_{x}-i \alpha_{y} k_{y}$ and $S_{k_{z}}=\alpha_{z} k_{z}$ are the spin-orbit fields with $\left\{\alpha_{x}, \alpha_{y}, \alpha_{z}\right\} \geqslant 0$. The eigenvalues of this Hamiltonian matrix are

$$
\varepsilon_{\mathbf{k}, s}=\epsilon_{\mathbf{k},+}+s \sqrt{\left(\epsilon_{\mathbf{k},-}+S_{k_{z}}\right)^{2}+\left|S_{\mathbf{k}_{\perp}}\right|^{2}},
$$

where $s= \pm$ labels the helicity bands, and $\epsilon_{\mathbf{k}, s}=\left(\epsilon_{\mathbf{k}, \uparrow}+\right.$ $\left.s \epsilon_{\mathbf{k}, \downarrow}\right) / 2$ are the kinetic energy average and half of the kinetic energy difference of $\uparrow$ and $\downarrow$ fermions. The corresponding eigenfunctions $\mathbf{u}_{s}^{\dagger}=\left(u_{1, s}^{*}, u_{2, s}^{*}\right)$ are given by $u_{1, s} / u_{2, s}=S_{\mathbf{k}_{\perp}} /\left[\epsilon_{\mathbf{k},-}+S_{k_{z}}-s \sqrt{\left(\epsilon_{\mathbf{k},-}+S_{k_{z}}\right)^{2}+\left|S_{\mathbf{k}_{\perp}}\right|^{2}}\right]$. Throughout this paper, we mainly consider four analytically tractable spin-orbit fields: (i) $\alpha_{x}=\alpha$ and $\alpha_{y}=\alpha_{z}=$ 0 corresponding to an equal mixture of Rashba-type [22] and Dresselhaus-type [23] SOCs (ERD), (ii) $\alpha_{z}=\alpha$ and $\alpha_{x}=\alpha_{y}=0$ corresponding to a fully aligned SOC (FA), (iii) $\alpha_{x}=\alpha_{y}=\alpha$ and $\alpha_{z}=0$ corresponding to a purely Rashba- or Dresselhaus-type SOC (PRD), and (iv) $\alpha_{x}=\alpha_{y}=$ $\alpha_{z}=\alpha$ corresponding to a fully spherical SOC (FS). We note that ERD- and FA-type SOCs are essentially the same for balanced Fermi gases, but these cases differ substantially for imbalanced Fermi gases, as we discuss below in Sec. III.

For the interacting SOC fermions, it has recently been shown that the strength of the attractive particle-particle interaction $g \geqslant 0$ is related to the two-body binding energy $\epsilon_{b} \leqslant 0$ in vacuum via $1 / g=(1 / 2) \sum_{\mathbf{k}, s} 1 /\left(2 \varepsilon_{\mathbf{k}, s}+\epsilon_{\mathrm{th}}-\epsilon_{b}\right)$, where $\epsilon_{\mathrm{th}}=m \alpha^{2}$ is the energy threshold for the two-body bound state. As usually done, the theoretical parameter $g$ can be eliminated in favor of the experimentally relevant $s$-wave scattering length $a_{s}$ via the relation $1 / g=-m V /\left(4 \pi a_{s}\right)+$ $\sum_{\mathbf{k}} 1 /\left(2 \epsilon_{\mathbf{k},+}\right)$, where $V$ is the volume.

The bound-state equation is analytically tractable for the four cases mentioned above. For the simplest ERD- and FA-type SOCs, a two-body bound state with energy $\epsilon_{b}=$ $-1 /\left(m a_{s}^{2}\right)$ exists only when $a_{s}>0$, showing that the ERDand FA-type SOCs do not have any observable effect on the two-body problem. On the other hand, in the case of

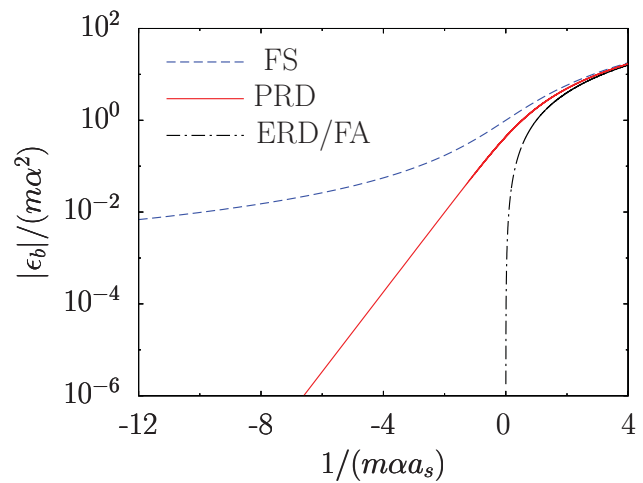

FIG. 1. (Color online) The binding energy $\epsilon_{b} \leqslant 0$ of the two-body bound state are shown as a function $1 /\left(m \alpha a_{s}\right)$ for the ERD-, FA-, PRD- and FS-type SOCs.

a PRD-type SOC, a two-body bound state exists even for $a_{s}<0$ [12], and its energy is determined by [14,19]

$$
\frac{1}{m \alpha a_{s}}=\sqrt{1-\frac{\epsilon_{b}}{m \alpha^{2}}}-\ln \left(\sqrt{1-\frac{m \alpha^{2}}{\epsilon_{b}}}+\sqrt{\frac{m \alpha^{2}}{-\epsilon_{b}}}\right) \text {. }
$$

In the weak SOC limit, when $m \alpha^{2} \ll\left|\epsilon_{b}\right|$, this expression gives $\epsilon_{b} \approx-1 /\left(m a_{s}^{2}\right)-m \alpha^{2}+2 \alpha / a_{s}$ up to the leading order in $\alpha$, and it recovers the usual result in the $\alpha \rightarrow 0$ limit. However, in the strong SOC limit, when $m \alpha^{2} \gg\left|\epsilon_{b}\right|$, Eq. (3) gives $\epsilon_{b} \approx$ $-\left(4 m \alpha^{2} / e^{2}\right) e^{2 /\left(m \alpha a_{s}\right)}$, which is exponentially small, as shown in Fig. 1. We also obtain $\epsilon_{b} \approx-0.44 m \alpha^{2}$ at unitarity $[14,15]$. Similarly, in the FS-type SOC, an even deeper two-body bound state exists for $a_{s}<0$, and its energy is given by

$$
\epsilon_{b}=-\frac{1}{2 m a_{s}^{2}}-m \alpha^{2} \pm \sqrt{\frac{1}{4 m^{2} a_{s}^{4}}+\frac{\alpha^{2}}{a_{s}^{2}}},
$$

where the $+(-)$ sign is valid for $a_{s}<0\left(a_{s}>0\right)$. This expression reduces to $\epsilon_{b} \approx-m^{3} \alpha^{4} a_{s}^{2}$ in the weak SOC limit and $\epsilon_{b} \approx-1 /\left(m a_{s}^{2}\right)-2 m \alpha^{2}$ in the strong SOC limits, and to $\epsilon_{b}=-m \alpha^{2}$ at unitarity.

Having shown that different types of SOCs give rise to significant differences with regard to the existence of the twobody bound state and its binding energy, next we analyze the many-body problem where these differences also play an important role.

\section{MANY-BODY PROBLEM}

Let us first consider noninteracting ( $g=0$ or $a_{s} \rightarrow 0^{-}$) balanced $\left(N_{\uparrow}=N_{\downarrow}=N / 2\right.$ or $\left.\mu_{\uparrow}=\mu_{\downarrow}=\mu\right)$ Fermi gases at zero temperature. For this purpose, and throughout this paper, we conveniently choose the energy (length) scale as the Fermi energy $\epsilon_{F}$ (Fermi momentum $k_{F}$ ) of $N / 2$ fermions such that $N=k_{F}^{3} V /\left(3 \pi^{2}\right)$.

It has been shown that increasing the SOC for a noninteracting Fermi gas leads to a change in the Fermi-surface topology, when the number of fermions in the + -helicity band $\left(N_{+}\right)$vanishes [13], where $N_{s}=\sum_{\mathbf{k}} \theta\left(\mu-\varepsilon_{\mathbf{k}, s}\right)$. This occurs when the chemical potential $\mu$ goes below the bottom of the energy band, i.e., when $\mu=0$, or when $\alpha$ increases beyond a critical value $\left(\alpha_{c}\right)$. In some ways, this is similar to the usual 
BCS-BEC crossover problem, where the quasiparticle and quasihole excitation spectrum changes behavior as a function of increasing the scattering parameter $1 /\left(k_{F} a_{s}\right)$ at $\mu=0$, i.e., its minimum is located at a finite (zero) momenta when $\mu>0$ $(\mu<0)$.

For the ERD- and FA-type SOCs, we obtain $\mu=\epsilon_{F}-m \alpha^{2} / 2$, and thus setting $\mu=0$ gives $\alpha_{c}=k_{F} / m$. For the PRD-type SOC, we obtain $m \sqrt{m} \sqrt{2 \mu}(2 \mu+$ $\left.9 m \alpha^{2} / 2\right)=k_{F}^{3}$ for $\mu \geqslant 0$, leading to $\mu \approx \epsilon_{F}-3 m \alpha^{2} / 2$ in the weak SOC limit when $m \alpha^{2} \ll \epsilon_{F}$, and also obtain $\mu=2 k_{F}^{3} /\left(3 \pi m^{2} \alpha\right)-m \alpha^{2} / 2$ for $\mu \leqslant 0$. Thus, setting $\mu=0$ gives $\alpha_{c}=[4 /(3 \pi)]^{1 / 3} k_{F} / m \approx 0.75 k_{F} / m$. Lastly, for the FS-type SOC, we obtain $2 m \sqrt{m}\left(\mu+2 m \alpha^{2}\right)$ $\sqrt{2 \mu+m \alpha^{2}}=k_{F}^{3}, \quad$ leading to $\mu=-3 m \alpha^{2} / 2+m^{3} \alpha^{4} /$ $\left[4\left(2 m^{6} \alpha^{6}+k_{F}^{6}+k_{F}^{3} \sqrt{4 m^{6} \alpha^{6}+k_{F}^{6}}\right)\right]^{1 / 3}+\left(m^{6} \alpha^{6}+k_{F}^{6} / 2+\right.$ $\left.k_{F}^{3} \sqrt{4 m^{6} \alpha^{6}+k_{F}^{6}} / 2\right)^{1 / 3} /(2 m)$. Thus, again setting $\mu=0$ gives $\alpha_{c}=(1 / 4)^{1 / 3} k_{F} / m \approx 0.63 k_{F} / m$.

We emphasize that the aforementioned change in Fermisurface topology for the noninteracting balanced Fermi gases is not a quantum phase transition but a smooth crossover $[13,19]$. Adding the interactions does not effect this picture much [13-16,19,21], and the crossover in the Fermi-surface topology again occurs at $\mu=0$. However, this is no longer the case for the interacting imbalanced Fermi gases [18-21], and next we show how this crossover picture becomes a quantum phase transition in the presence of a population imbalance within the self-consistent mean-field theory.

\section{A. Mean-field theory}

In the absence of a SOC and at low temperatures, it is well established that the mean-field theory is sufficient to describe the Fermi gases both in the BCS and the BEC limits, and that this theory also captures qualitatively the correct physics in the entire BCS-BEC evolution [1,2]. Hoping that the mean-field formalism remains sufficient in the presence of a SOC, here we analyze the resultant ground-state phase diagrams.

For this purpose, we use the mean-field Hamiltonian

$$
\begin{aligned}
H= & \frac{1}{2} \sum_{\mathbf{k}} \psi_{\mathbf{k}}^{\dagger} \\
& \times\left(\begin{array}{cccc}
\xi_{\mathbf{k}, \uparrow}+S_{k_{z}} & S_{\mathbf{k}_{\perp}} & 0 & \Delta \\
S_{\mathbf{k}_{\perp}}^{*} & \xi_{\mathbf{k}, \downarrow}-S_{k_{z}} & -\Delta & 0 \\
0 & -\Delta^{*} & -\xi_{\mathbf{k}, \uparrow}+S_{k_{z}} & S_{\mathbf{k}_{\perp}}^{*} \\
\Delta^{*} & 0 & S_{\mathbf{k}_{\perp}} & -\xi_{\mathbf{k}, \downarrow}-S_{k_{z}}
\end{array}\right) \\
& \times \psi_{\mathbf{k}}+\sum_{\mathbf{k}} \xi_{\mathbf{k},+}+\frac{|\Delta|^{2}}{g},
\end{aligned}
$$

where $\psi_{\mathbf{k}}^{\dagger}=\left[a_{\mathbf{k}, \uparrow}^{\dagger}, a_{\mathbf{k}, \downarrow}^{\dagger}, a_{-\mathbf{k}, \uparrow}, a_{-\mathbf{k}, \downarrow}\right]$ denotes the fermionic operators collectively, $\xi_{\mathbf{k}, \sigma}=\epsilon_{\mathbf{k}, \sigma}-\mu_{\sigma}$, and $\Delta=g\left\langle a_{\mathbf{k}, \uparrow} a_{-\mathbf{k}, \downarrow}\right\rangle$ is the mean-field order parameter where $\langle\cdots\rangle$ is the thermal average. The mean-field thermodynamic potential can be written as [8]

$$
\Omega=\frac{T}{2} \sum_{\mathbf{k}, \lambda} \ln \left(\frac{1+X_{\mathbf{k}, \lambda}}{2}\right)+\sum_{\mathbf{k}} \xi_{\mathbf{k},+}+\frac{|\Delta|^{2}}{g},
$$

where $T$ is the temperature, $\lambda=\{1,2,3,4\}$ labels the quasiparticle and quasihole excitation energies $E_{\mathbf{k}, \lambda}, X_{\mathbf{k}, \lambda}=$ $\tanh \left[E_{\mathbf{k}, \lambda} /(2 T)\right]$, and $\xi_{\mathbf{k}, s}=\epsilon_{\mathbf{k}, s}-\mu_{s}$, where $\mu_{s}=\left(\mu_{\uparrow}+\right.$ $\left.s \mu_{\downarrow}\right) / 2$. Here, the quasiparticle and quasihole excitation energies $E_{\mathbf{k}, \lambda}$ are determined by the eigenvalues of the Hamiltonian matrix given in Eq. (5). Following the usual procedure, i.e., $\partial \Omega / \partial|\Delta|=0$ for the order parameter and $N_{\uparrow}+s N_{\downarrow}=-\partial \Omega / \partial \mu_{s}$ for the number equations, we obtain the self-consistency equations

$$
\begin{gathered}
\frac{2|\Delta|}{g}=\frac{1}{4} \sum_{\mathbf{k}, \lambda} \frac{\partial E_{\mathbf{k}, \lambda}}{\partial|\Delta|}\left(X_{\mathbf{k}, \lambda}-1\right), \\
N_{\uparrow} \pm N_{\downarrow}=\frac{1}{4} \sum_{\mathbf{k}, \lambda}\left[\frac{1 \pm 1}{2}+\frac{\partial E_{\mathbf{k}, \lambda}}{\partial \mu_{ \pm}}\left(X_{\mathbf{k}, \lambda}-1\right)\right] .
\end{gathered}
$$

These equations are the generalization of the mean-field order parameter and number equations to the case of an anisotropic SOC, and they are consistent with the known results in the appropriate limits [13-16,18,19,21] (see Sec. III B).

As usual, we checked the stability of the mean-field solutions for the uniform superfluid phase using the curvature criterion [19,21], which says that the curvature of $\Omega$ with respect to $|\Delta|$, i.e.,

$$
\begin{aligned}
\frac{\partial^{2} \Omega}{\partial|\Delta|^{2}}= & \frac{1}{4} \sum_{\mathbf{k}, \lambda}\left[\left(\frac{1}{|\Delta|} \frac{\partial E_{\mathbf{k}, \lambda}}{\partial|\Delta|}-\frac{\partial^{2} E_{\mathbf{k}, \lambda}}{\partial|\Delta|^{2}}\right)\left(X_{\mathbf{k}, \lambda}-1\right)\right. \\
& \left.-\frac{1}{2 T}\left(\frac{\partial E_{\mathbf{k}, \lambda}}{\partial|\Delta|}\right)^{2} Y_{\mathbf{k}, \lambda}\right],
\end{aligned}
$$

needs to be positive, where $Y_{\mathbf{k}, \lambda}=\operatorname{sech}^{2}\left[E_{\mathbf{k}, \lambda} /(2 T)\right]$. When the curvature $\partial^{2} \Omega / \partial|\Delta|^{2}$ is negative, the uniform mean-field solution does not correspond to a minimum of $\Omega$, and a nonuniform superfluid phase, e.g., a phase separation, is favored. It is known that the curvature criterion correctly discards the unstable solutions, but metastable solutions may still survive. This may cause minor quantitative changes in the first-order phase transition boundaries [20].

\section{B. Quasiparticle and quasihole excitations}

While the eigenvalues of the Hamiltonian matrix given in Eq. (5) do not acquire a simple analytic form for a general SOC, next we discuss three limits where the quasiparticle and quasihole excitation energies simplify considerably, allowing for further analytical investigation.

\section{Balanced Fermi gases with $S_{\mathrm{k}_{\perp}} \neq 0$ and $S_{k_{z}} \neq 0$}

First of all, for balanced $\left(\xi_{\mathbf{k}, \uparrow}=\xi_{\mathbf{k}, \downarrow}\right)$ Fermi gases, we obtain [13-17,19,22]

$$
E_{\mathbf{k}, \lambda}=s_{\lambda} \sqrt{\left(\xi_{\mathbf{k},+}+p_{\lambda} \sqrt{\left|S_{\mathbf{k}_{\perp}}\right|^{2}+S_{k_{z}}^{2}}\right)^{2}+|\Delta|^{2}},
$$

where $s_{1}=s_{2}=+$ and $s_{3}=s_{4}=-$, and $p_{\lambda}=-(-1)^{\lambda}$, i.e., $p_{1}=p_{3}=+$ and $p_{2}=p_{4}=-$. Since all $E_{\mathbf{k}, \lambda}$ have no zeros and are always gapped for all parameters in $\mathbf{k}$ space, we expect the BCS-BEC evolution to be a smooth crossover for balanced Fermi gases even in the presence of a SOC [13-16,19]. This is similar to what happens in the usual BCS-BEC crossover picture of a Fermi gas with no SOC, where the quasiparticle 
and quasihole excitation energies are also gapped. Therefore, the ground state of balanced Fermi gases with SOC is a topologically trivial superfluid [19-21].

In this case, the order parameter equation reduces to $1 / g=(1 / 2) \sum_{\mathbf{k}, s} X_{\mathbf{k}, s} /\left(2 E_{\mathbf{k}, s}\right)$ and the number equation reduces to $N=(1 / 2) \sum_{\mathbf{k}, s}\left[1-\left(\xi_{\mathbf{k}, s} / E_{\mathbf{k}, s}\right) X_{\mathbf{k}, s}\right]$, where $E_{\mathbf{k},+(-)}=E_{\mathbf{k}, 1(2)}[13-17,22]$. These equations are analytically tractable in the strong-coupling limit when $\mu<0$ and $|\mu| \gg|\Delta|$, for which they are approximately given by $1 / g \approx(1 / 2) \sum_{\mathbf{k}, s} 1 /\left[2\left(\varepsilon_{\mathbf{k}, s}-\mu\right)\right]$ and $N \approx\left(|\Delta|^{2} / 2\right) \sum_{\mathbf{k}, s}$ $1 /\left[2\left(\varepsilon_{\mathbf{k}, s}-\mu\right)^{2}\right]$, respectively, where $\varepsilon_{\mathbf{k}, s}$ is given in Eq. (2). Comparing the order parameter equation with the bound state, one immediately leads to $\mu=\left(\epsilon_{b}-m \alpha^{2}\right) / 2$ for all four types of SOC, but the number equation leads to $|\Delta|^{2}=$ $16 \sqrt{\epsilon_{F}^{3}\left(2|\mu|-m \alpha^{2}\right)} /(3 \pi)$ for the ERD- and FA-type SOCs, $|\Delta|^{2}=2 \sqrt{2} k_{F}^{3}\left(2|\mu|-m \alpha^{2}\right) /(3 \pi m \sqrt{m|\mu|})$ for the PRD-type SOC, and $|\Delta|^{2}=2 k_{F}^{3}\left(2|\mu|-m \alpha^{2}\right)^{3 / 2} /(3 \pi m \sqrt{m}|\mu|)$ for the FS-type SOC. We note that $|\Delta|$ is independent of $\alpha$ only in the ERD- and FA-type SOCs, which is consistent with the recent numerical findings $[13,16]$. This is not surprising since the SOC term can be eliminated by a momentum shift in the $x$ and $z$ directions, respectively, in the ERD- and FA-type SOCs, which also leads to a shift in the chemical potential $\mu(\alpha)=\mu(0)-m \alpha^{2} / 2$. Since Eq. (10) depends only on the total magnitude of the SOC, i.e., $\sqrt{\left|S_{\mathbf{k}_{\perp}}\right|^{2}+S_{k_{z}}^{2}}$, the ERD-, FA-, PRD-, and FS-type SOCs differ in their Jacobians of the k-space integrals.

\section{2. $S_{\mathrm{k}_{\perp}} \rightarrow 0$ and $S_{k_{z}} \neq 0$}

When $S_{\mathbf{k}_{\perp}} \rightarrow 0$ and $S_{k_{z}} \neq 0$, i.e., the FA-type SOC, we obtain

$$
E_{\mathbf{k}, \lambda}=s_{\lambda} \sqrt{\left(\xi_{\mathbf{k},+}+p_{\lambda} S_{k_{z}}\right)^{2}+|\Delta|^{2}}+p_{\lambda} \xi_{\mathbf{k},-},
$$

which again can be gapless in some $\mathbf{k}$-space regions. The zeros of $E_{\mathbf{k}, \lambda}$ can be found by imposing the condition $E_{\mathbf{k}, 1(2)} E_{\mathbf{k}, 3(4)}=$ $\xi_{\mathbf{k},-}^{2}-\left(\xi_{\mathbf{k},+}+p_{\lambda} S_{k_{z}}\right)^{2}-|\Delta|^{2}=0$, indicating that the zeros occur at real $\left(k_{\perp}=\sqrt{k_{x}^{2}+k_{y}^{2}}, k_{z}\right)$ momenta such that $k_{\perp, s}^{2}=2 m \mu_{+}-k_{z, s}^{2}+2 m S_{k_{z, s}}+2 m s \sqrt{\mu_{-}^{2}-|\Delta|^{2}}$. Since $k_{\perp, s} \geqslant 0$, setting $k_{\perp, s}=0$ above leads to $k_{z, s}=m \alpha \pm$ $\sqrt{2 m\left(\mu_{+}+m \alpha^{2} / 2\right)+2 m s \sqrt{\mu_{-}^{2}-|\Delta|^{2}}}$, provided that $|\Delta|<$ $\left|\mu_{-}\right|$and $|\Delta|^{2}<-\left(\mu_{\uparrow}+m \alpha^{2} / 2\right)\left(\mu_{\downarrow}+m \alpha^{2} / 2\right)$. This analysis shows that the conditions $|\Delta|=\left|\mu_{-}\right|$and $|\Delta|^{2}=-\left(\mu_{\uparrow}+\right.$ $\left.m \alpha^{2} / 2\right)\left(\mu_{\downarrow}+m \alpha^{2} / 2\right)$ determine the phase boundaries between the SF, GSF(I), and GSF(II) regions (see Sec. III C), and that these three phases meet at a tricritical point determined by $\mu_{+}=-m \alpha^{2} / 2$.

In this case, the derivatives of the quasiparticle and quasihole energies are given by $\partial E_{\mathbf{k}, \lambda} / \partial|\Delta|=s_{\lambda}|\Delta| / B_{\mathbf{k}, p_{\lambda}}$ for the order parameter, $\partial E_{\mathbf{k}, \lambda} / \partial \mu_{+}=-s_{\lambda}\left(\xi_{\mathbf{k},+}+p_{\lambda} S_{k_{z}}\right) / B_{\mathbf{k}, p_{\lambda}}$ for the average chemical potential, and $\partial E_{\mathbf{k}, \lambda} / \partial \mu_{-}=$ $-p_{\lambda}$ for the half of the chemical potential difference. Here, $B_{\mathbf{k}, p_{\lambda}}=\sqrt{\left(\xi_{\mathbf{k},+}+p_{\lambda} S_{k_{z}}\right)^{2}+|\Delta|^{2}}$. We again note for this case that the order parameter equation reduces to $2|\Delta| / g=(1 / 4) \sum_{\mathbf{k}, \lambda}\left(\partial E_{\mathbf{k}, \lambda} / \partial|\Delta|\right) X_{\mathbf{k}, \lambda}$, and the number equations reduce to $N_{\uparrow} \pm N_{\downarrow}=(1 / 4) \sum_{\mathbf{k}, \lambda}[(1 \pm 1) / 2+$ $\left.\left(\partial E_{\mathbf{k}, \lambda} / \partial \mu_{ \pm}\right) X_{\mathbf{k}, \lambda}\right]$.

Similar to the balanced case discussed in Sec. III B 1, we note that the FA-type SOC term again can be eliminated for the population-imbalanced Fermi gases, by a momentum shift in the $z$ direction [24]. This also leads to a shift in the chemical potentials, i.e., $\mu_{\sigma}(\alpha)=\mu_{\sigma}(0)-m \alpha^{2} / 2$. Therefore, the FAtype SOC does not have any observable effect on the phase diagrams, which is in sharp contrast with the ERD-type SOC, as discussed next.

\section{3. $S_{k_{z}} \rightarrow 0$ and $S_{\mathrm{k}_{\perp}} \neq 0$}

On the other hand, when $S_{k_{z}} \rightarrow 0$ and $S_{\mathbf{k}_{\perp}} \neq 0$, it is straightforward to show that $[8,18-21]$

$$
E_{\mathbf{k}, \lambda}=s_{\lambda} \sqrt{\xi_{\mathbf{k},+}^{2}+\xi_{\mathbf{k},-}^{2}+|\Delta|^{2}+\left|S_{\mathbf{k}_{\perp}}\right|^{2}+2 p_{\lambda} A_{\mathbf{k}}},
$$

where $A_{\mathbf{k}}=\sqrt{\xi_{\mathbf{k},-}^{2}\left(\xi_{\mathbf{k},+}^{2}+|\Delta|^{2}\right)+\left|S_{\mathbf{k}_{\perp}}\right|^{2} \xi_{\mathbf{k},+}^{2}}$. Note in this case that $E_{\mathbf{k}, \lambda}$ can be gapless at some points and lines in $\mathbf{k}$ space. The zeros of $E_{\mathbf{k}, \lambda}$ can be found by imposing the condition $E_{\mathbf{k}, 1(3)}^{2} E_{\mathbf{k}, 2(4)}^{2}=\left(\xi_{\mathbf{k},+}^{2}-\xi_{\mathbf{k},-}^{2}+|\Delta|^{2}-\right.$ $\left.\left|S_{\mathbf{k}_{\perp}}\right|^{2}\right)^{2}+4|\Delta|^{2}\left|S_{\mathbf{k}_{\perp}}\right|^{2}=0$, indicating that both $\left|S_{\mathbf{k}_{\perp}}\right|=0$ and $\xi_{\mathbf{k}, \uparrow} \xi_{\mathbf{k}, \downarrow}+|\Delta|^{2}=0$ needs to be satisfied. Therefore, in the PRD-type SOC, the zeros occur when $[19,21] k_{x}=k_{y}=0$ and at real $k_{z}$ momenta, $k_{z, s}^{2}=2 m \mu_{+}+2 m s \sqrt{\mu_{-}^{2}-|\Delta|^{2}}$, provided that $|\Delta|<\left|\mu_{-}\right|$for $\mu_{+} \geqslant 0$, and $|\Delta|^{2}<-\mu_{\uparrow} \mu_{\downarrow}$ for $\mu_{+}<0$. Similarly, in the ERD-type SOC, the zeros occur when $k_{x}=0$ and at real $k_{\rho}=\sqrt{k_{y}^{2}+k_{z}^{2}}$ momenta, $k_{\rho, s}^{2}=$ $2 m \mu_{+}+2 m s \sqrt{\mu_{-}^{2}-|\Delta|^{2}}$, provided with the same conditions as above. This analysis shows for both PRD- and ERD-type SOCs that the conditions $|\Delta|=\left|\mu_{-}\right|$and $|\Delta|^{2}=-\mu_{\uparrow} \mu_{\downarrow}$ determine the phase boundaries between the SF, GSF(I), and GSF(II) regions (see Sec. III C), such that these three phases meet at a tricritical point determined by $\mu_{+}=0$ [19-21]. In Fig. 2, typical excitation spectra $E_{\mathbf{k}, \lambda}$ of the PRD-type SOC are shown for the SF, GSF(I), and GSF(II) phases, illustrating the k-space topology of their gapped or gapless excitations as discussed in Sec. III C in great detail.

In this case, the derivatives of the quasiparticle and quasihole energies are given by $\partial E_{\mathbf{k}, \lambda} / \partial|\Delta|=(1+$ $\left.p_{\lambda} \xi_{\mathbf{k},-}^{2} / A_{\mathbf{k}}\right)|\Delta| / E_{\mathbf{k}, \lambda}$ for the order parameter, $\partial E_{\mathbf{k}, \lambda} / \partial \mu_{+}=$ $-\left[1+p_{\lambda}\left(\xi_{\mathbf{k},-}^{2}+\left|S_{\mathbf{k}_{\perp}}\right|^{2}\right) / A_{\mathbf{k}}\right] \xi_{\mathbf{k},-} / E_{\mathbf{k}, \lambda}$ for the average chemical potential, and $\partial E_{\mathbf{k}, \lambda} / \partial \mu_{-}=-\left[1+p_{\lambda}\left(\xi_{\mathbf{k},+}^{2}+|\Delta|^{2}\right) /\right.$ $\left.A_{\mathbf{k}}\right] \xi_{\mathbf{k},-} / E_{\mathbf{k}, \lambda}$ for the half of the chemical potential difference. Therefore, the order parameter equation reduces to $2|\Delta| / g=(1 / 2) \sum_{\mathbf{k}, s}\left(\partial E_{\mathbf{k}, s} / \partial|\Delta|\right) X_{\mathbf{k}, s}$, and the number equations reduce to $N_{\uparrow} \pm N_{\downarrow}=(1 / 2) \sum_{\mathbf{k}, s}[(1 \pm 1) / 2+$ $\left.\left(\partial E_{\mathbf{k}, s} / \partial \mu_{ \pm}\right) X_{\mathbf{k}, s}\right]$, where $E_{\mathbf{k},+(-)}=E_{\mathbf{k}, 1(2)}[19,21]$.

\section{Ground-state phase diagrams}

There are three phases in the phase diagrams [19-21]. While the normal $(\mathrm{N})$ phase is characterized by $\Delta=0$, the uniform superfluid and nonuniform superfluid, e.g., phase separation (PS), are characterized by $\partial^{2} \Omega / \partial|\Delta|^{2}>0$ and $\partial^{2} \Omega / \partial|\Delta|^{2}<$ 0 , respectively, when $\Delta \neq 0$. Furthermore, in addition to the 

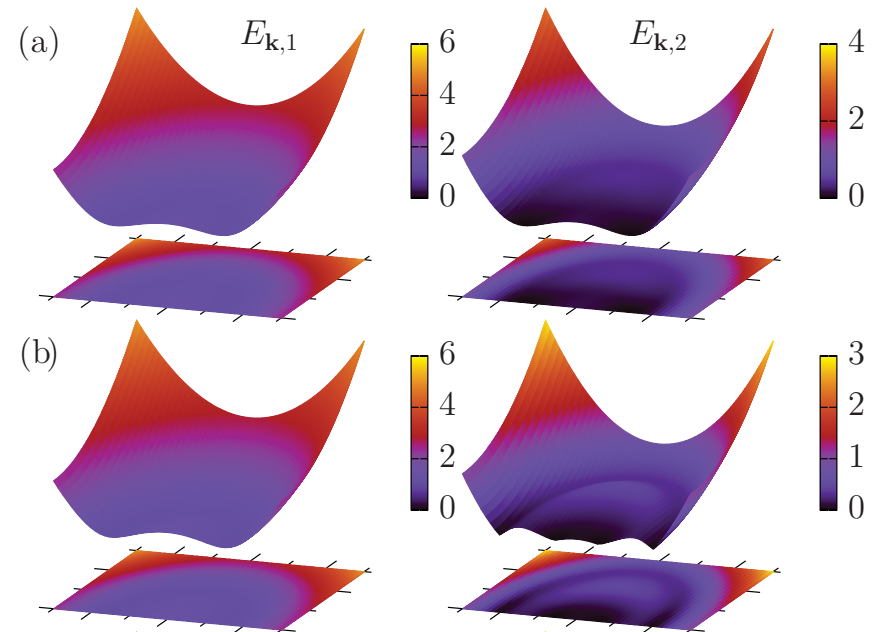

(c)

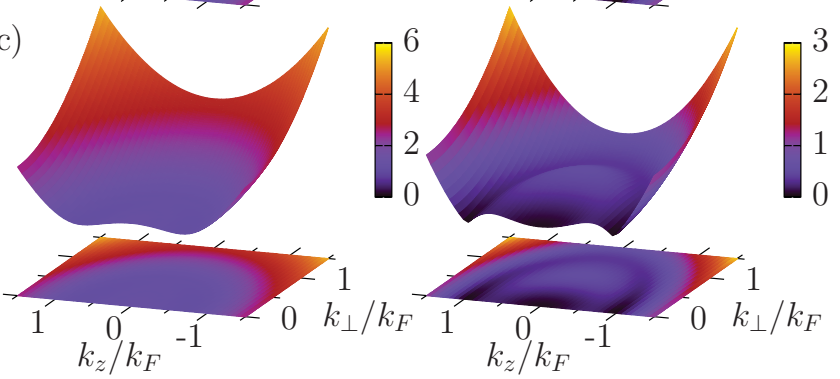

FIG. 2. (Color online) The excitation spectra $E_{\mathbf{k}, \lambda}$ of the PRDtype SOC are shown for (a) the SF phase $\left(\alpha=0.275 k_{F} / m\right.$ and $P=0.25)$, (b) the GSF(II) phase $\left(\alpha=0.275 k_{F} / m\right.$ and $\left.P=0.5\right)$, and (c) the GSF(I) phase $\left(\alpha=0.35 k_{F} / m\right.$ and $\left.P=0.5\right)$. These data correspond to the red cross marks in Fig. 4(b).

topologically trivial gapped superfluid (SF) phase, the gapless superfluid (GSF) phase can also be distinguished by the momentum-space topology of its excitations. Depending on the number of zeros of $E_{\mathbf{k}, \lambda}$ (zero-energy regions in $\mathbf{k}$ space), there are two topologically distinct gapless phases. For the ERD-type SOC, we have GSF(I), where $E_{\mathbf{k}, \lambda}$ has one, and $\mathrm{GSF}(\mathrm{II})$, where $E_{\mathbf{k}, \lambda}$ has two zero-energy rings in $\mathbf{k}$ space. Similarly, for the PRD-type SOC, we have GSF(I), where $E_{\mathbf{k}, \lambda}$ has two, and GSF(II), where $E_{\mathbf{k}, \lambda}$ has four zero-energy points in $\mathbf{k}$ space. The topological classification of uniform superfluid phases are summarized in Table I. In Fig. 2, we show the excitation spectra $E_{\mathbf{k}, \lambda}$ of the PRD-type SOC for the SF phase in Fig. 2(a), GSF(II) phase in Fig. 2(b), and GSF(I) phase in Fig. 2(c), illustrating k-space topology of their gapped or gapless excitations. These data correspond to the points indicated by the red cross marks in Fig. 4(b).

TABLE I. The topological classification of uniform superfluid phases are summarized, depending on the number of zero-energy quasiparticle and quasihole excitation energy surfaces, rings, or points in $\mathbf{k}$ space.

\begin{tabular}{lcccc}
\hline \hline & No-SOC & Balanced & ERD & PRD \\
\hline SF & Gapped & Gapped & Gapped & Gapped \\
GSF(I) & 1 surface & N/A & 1 ring & 2 points \\
GSF(II) & 2 surfaces & N/A & 2 rings & 4 points \\
\hline \hline
\end{tabular}
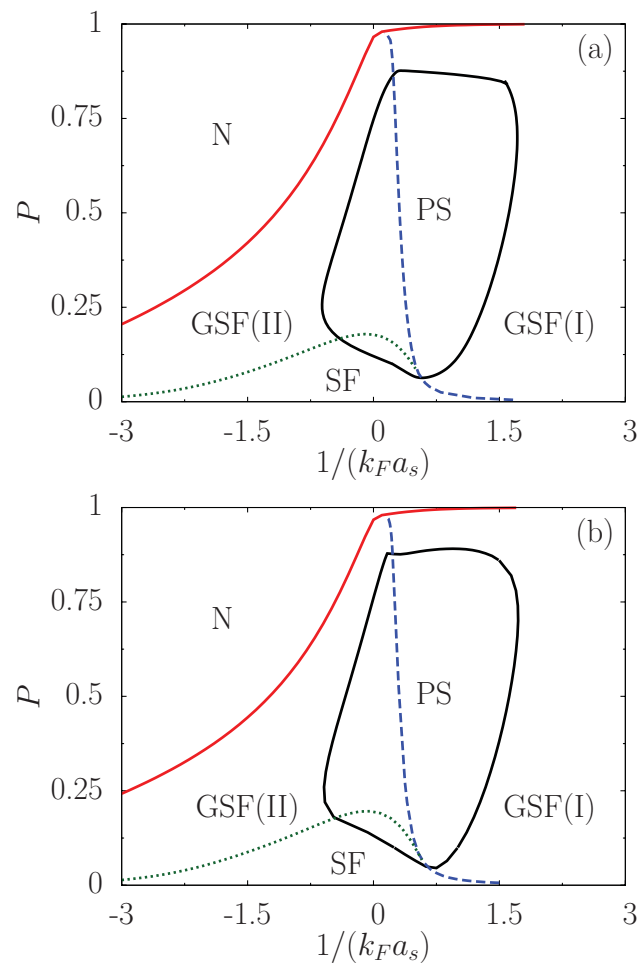

FIG. 3. (Color online) The ground-state phase diagrams of (a) ERD-type and (b) PRD-type SOC Fermi gases are shown as a function of $P=\left(N_{\uparrow}-N_{\downarrow}\right) / N$ and $1 /\left(k_{F} a_{s}\right)$. Here, we choose $\alpha=0.15 k_{F} / m$ in (a) and $\alpha=(0.15 / \sqrt{2}) k_{F} / m$ in (b), so that the magnitudes of the SOC are the same in both figures. The phase labels are described in the text (see Sec. III C). Note that the regions bounded by the solid black lines show instability toward a nonuniform superfluid phase (PS), and the dashed and dotted lines shown within these regions are solely for illustration purposes.

In Fig. 3, we show the ground-state phase diagrams of (a) ERD-type and (b) PRD-type SOC Fermi gases as a function of the population imbalance $P=\left(N_{\uparrow}-N_{\downarrow}\right) / N$ and the scattering parameter $1 /\left(k_{F} a_{s}\right)$. Here, the dashed blue and dotted green lines correspond to $|\Delta|^{2}=-\mu_{\uparrow} \mu_{\downarrow}$ and $|\Delta|=\left|\mu_{-}\right|$, respectively, and they mark the SF, GSF(I), and GSF(II) phase boundaries. We note that the regions bounded by the solid black lines show instability toward a nonuniform superfluid phase (PS). Since our classification of distinct topological phases applies only to the uniform superfluid region, the dashed and dotted lines shown within these regions are solely for illustration purposes.

We find that while the ERD-type SOC does not have any observable effect on the balanced Fermi gases (see Sec. III B 1), it gives rise to a phase diagram with very similar topological structure as that of the PRD-type SOC. This is not very surprising since the main difference between the ERDand PRD-type SOC is the Jacobians involved in the $\mathbf{k}$-space integrals. This must be contrasted with the FA-type SOC, which does not have any observable effect on the system even in the presence of a population imbalance (see Sec. III B 2), and therefore its phase diagram is exactly the same as that of the usual population-imbalanced Fermi gases without the SOC. Since this problem is well studied in the literature [1,2], we do not discuss it any further. 

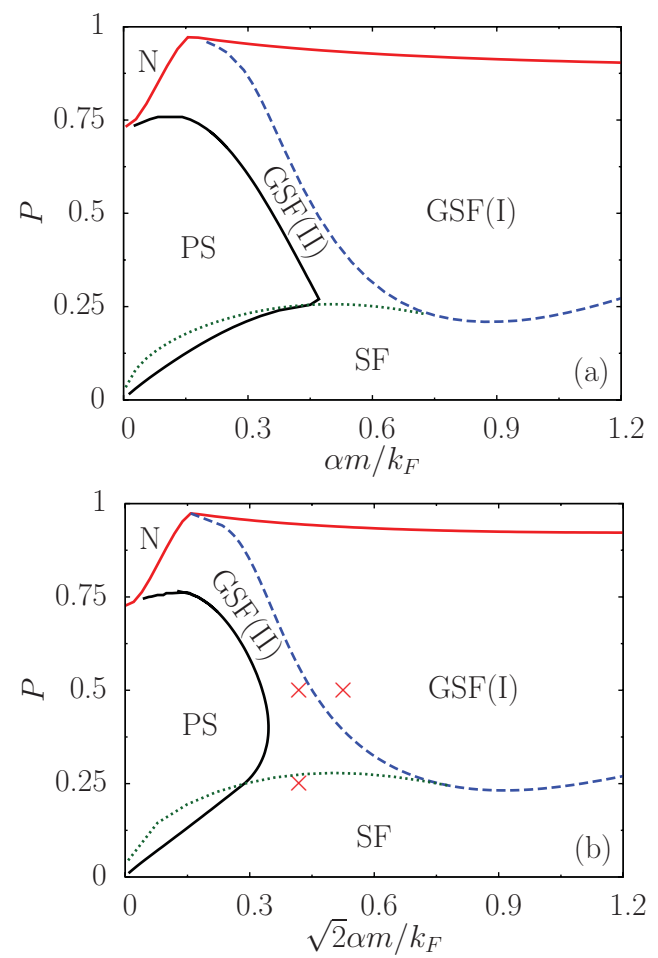

FIG. 4. (Color online) The ground-state phase diagrams of (a) ERD-type and (b) PRD-type SOCs are shown as a function of $P=\left(N_{\uparrow}-N_{\downarrow}\right) / N$ and $\alpha$ at unitarity, i.e., when $1 /\left(k_{F} a_{s}\right)=0$. The phase labels are described in the text (see Sec. IIIC). Note that the regions bounded by the solid black lines show instability toward a nonuniform superfluid phase (PS), and the dashed and dotted lines shown within these regions are solely for illustration purposes. In (b) the red crosses mark a point from each phase for which the corresponding excitation spectra and momentum distributions are shown in Figs. 2 and 5, respectively.

Comparing Fig. 3 with the $\alpha \rightarrow 0$ limit [1,2], it is clearly seen that both the ERD- and PRD-type SOCs are counteracting the population imbalance. On the one hand, this competition always tends to stabilize the GSF phase against the PS, and therefore, at any given $P$, the system eventually transitions to a stable SF or GSF by increasing $\alpha$, no matter how small is $1 /\left(k_{F} a_{s}\right)$. This is best seen in Fig. 4 , where the phase diagrams are shown as a function of $P$ and $\alpha$ at unitarity when $1 /\left(k_{F} a_{s}\right)=0$. On the other hand, we find that while both the ERD- and PRD-type SOCs stabilize the GSF phase against the $\mathrm{N}$ phase for low $P$ due to increased density of states $[13,14]$, they destabilize the GSF phase against the $\mathrm{N}$ phase for high $P$.

In sharp contrast to the $\alpha=0$ case where only the gapless GSF phase can support population imbalance, one of the intriguing effects of the ERD- and PRD-type SOCs is that both the gapless GSF and gapped SF phases can support population imbalance when $\alpha \neq 0$. This is possible due to the anisotropic nature of the SOC Fermi gases in $\mathbf{k}$ space. In fact, it has recently been shown for the mass-imbalanced Fermi gases that both the gapped SF and gapless GSF phases can support population balance when $\alpha \neq 0$ [21]. This is again in sharp contrast to the $\alpha=0$ case, where only the gapped SF phase can support population balance, and it is possible solely

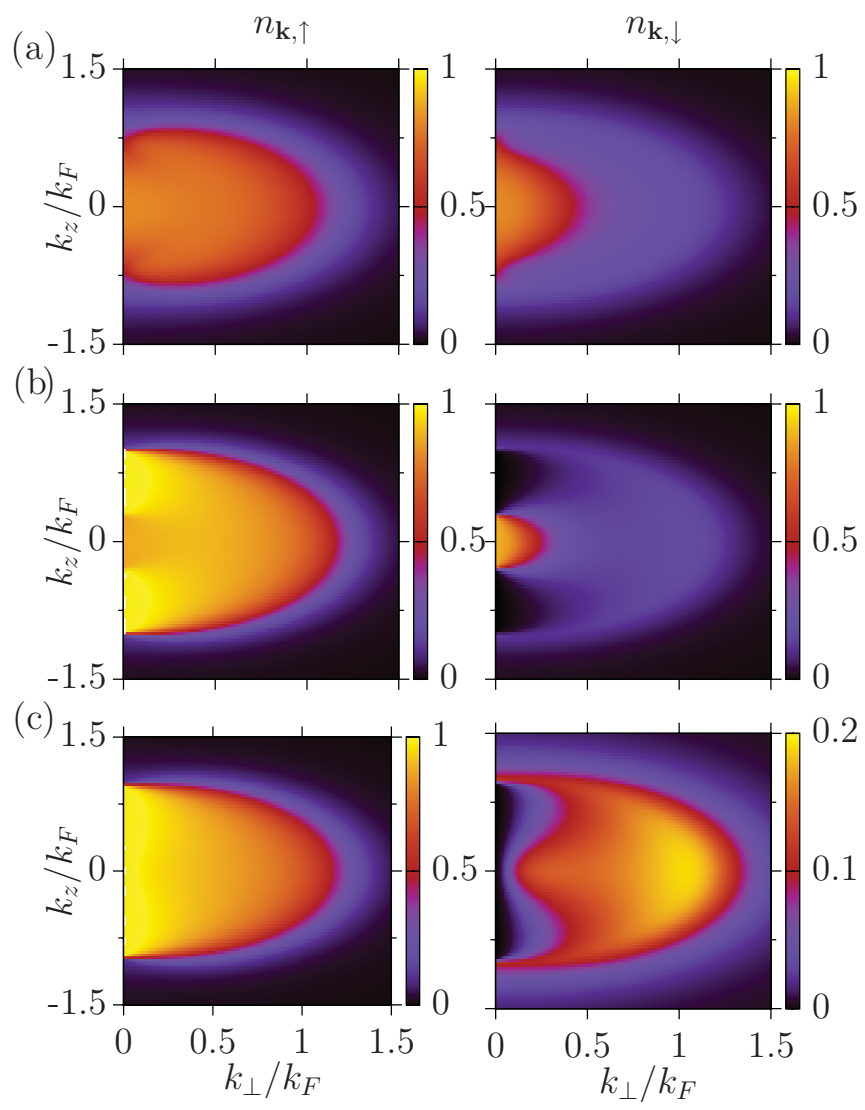

FIG. 5. (Color online) The momentum distributions $n_{\mathbf{k}, \sigma}$ of the PRD-type SOC are shown for (a) the SF phase, (b) the GSF(II) phase, and (c) the GSF(I) phase. These data again correspond to the points indicated by red cross marks in Fig. 4(b).

due to the anisotropic nature of the SOC Fermi gases in $\mathbf{k}$ space.

The transition from GSF(II) to GSF(I) leads to a change in topology in the lowest quasiparticle band, similar to the Lifshitz transition in ordinary metals and nodal (non- $s$-wave) superfluids. However, the topological transition discussed here is unique, because it involves an $s$-wave superfluid, and could be potentially observed through the measurement of the momentum distributions of $\uparrow$ and $\downarrow$ fermions $[19,20]$. The momentum distributions are readily available from Eq. (8), and we illustrate the typical $T=0$ distributions of the SF, GSF(I), and GSF(II) phases in Fig. 5. The distributions are anisotropic in $\mathbf{k}$ space, which follows from the anisotropic structure of $E_{\mathbf{k}, \lambda}$. In addition, while the distributions of SF phase do not show sharp features, those of GSF(I) and GSF(II) phases are exactly $n_{\mathbf{k}, \uparrow}=1$ and $n_{\mathbf{k}, \downarrow}=0$ for $\mathbf{k}$-space regions, where $k_{\perp}=0$ and $k_{z,-} \leqslant$ $\left|k_{z}\right| \leqslant k_{z,+}$. Therefore, a major redistribution occurs for the minority component $\left(n_{\mathbf{k}, \downarrow}\right)$ at the topological phase transition boundaries. For instance, at the GFS(II)-to-GSF(I) transition boundary, the sharp peak that is present near the origin vanishes abruptly.

Although this topological transition is quantum in its nature, signatures of it should still be observed at finite $T$, where the observables are smeared out due to thermal effects. While the primary signature of this topological transition is 
seen in the momentum distribution, single-particle spectral function [25] as well as some thermodynamic quantities such as the atomic compressibility would also show an anomaly at the transition boundary.

All of these results are obtained within the self-consistent mean-field approximation, which is known to be reliable for the entire BCS-BEC evolution only near $T=0$. Since the fluctuations dominate the physics at finite $T$ toward the molecular BEC limit [26], to emphasize further the effects of finite $T$, next we discuss the Gaussian fluctuations near the critical temperature $T_{c}$, i.e., the temperature at which the mean-field order parameter $\Delta$ vanishes.

\section{GAUSSIAN FLUCTUATIONS NEAR $\boldsymbol{T}_{\boldsymbol{c}}$}

One way to go beyond the mean-field (or saddle-point) approximation and study the Gaussian fluctuations is to use the imaginary-time functional integral approach [26,27]. Using this approach and expanding the order parameter field around $\Delta=0$, one obtains the fluctuation action $S_{G}=$ $(1 / T) \sum_{\mathbf{q}, n} L^{-1}\left(\mathbf{q}, v_{n}\right)\left|\Lambda\left(\mathbf{q}, v_{n}\right)\right|^{2}$, where $L^{-1}\left(\mathbf{q}, v_{n}\right)$ is the inverse fluctuation propagator, $\Lambda\left(\mathbf{q}, v_{n}\right)$ is the fluctuation field, and $v_{n}=2 \pi T n$ is the bosonic Matsubara frequency. It is a straightforward task to calculate the propagator

$$
\begin{aligned}
& L^{-1}\left(\mathbf{q}, v_{n}\right) \\
& =\frac{1}{g}-\frac{1}{8} \sum_{\mathbf{k}, \lambda_{o}, \lambda_{e}} \frac{X_{\mathbf{k}+\frac{\mathbf{q}}{2}, \lambda_{o}}-X_{\mathbf{k}-\frac{\mathbf{q}}{2}, \lambda_{e}}}{E_{\mathbf{k}+\frac{\mathbf{q}}{2}, \lambda_{o}}-E_{\mathbf{k}-\frac{\mathbf{q}}{2}, \lambda_{e}}-i v_{n}}\left[1-s_{\lambda_{o}} p_{\lambda_{e}}\right. \\
& \quad \times \frac{C_{\mathbf{k}+\frac{\mathbf{q}}{2},+} C_{\mathbf{k}-\frac{\mathbf{q}}{2},-}-\left(S_{\left.\mathbf{k}_{\perp}+\frac{\mathbf{q}_{\perp}}{2} S_{\mathbf{k}_{\perp}-\frac{\mathbf{q} \perp}{2}}^{*}+\text { H.c. }\right) / 2} \sqrt{\left(C_{\mathbf{k}+\frac{\mathbf{q}}{2},+}^{2}+\left|S_{\mathbf{k}_{\perp}+\frac{\mathbf{q}_{\perp}}{2}}\right|^{2}\right)\left(C_{\mathbf{k}-\frac{\mathbf{q}}{2},-}^{2}+\left|S_{\mathbf{k}_{\perp}-\frac{\mathbf{q}_{\perp}}{2}}\right|^{2}\right)}\right]}{},
\end{aligned}
$$

where $\lambda_{o}=\{1,3\}$ and $\lambda_{e}=\{2,4\}$ sums over odd and even $\lambda$ values, respectively, H.c. is the Hermitian conjugate, $C_{\mathbf{k}, s}=$ $\xi_{\mathbf{k},-}+s S_{k_{z}}$, and

$$
E_{\mathbf{k}, \lambda}=s_{\lambda} \sqrt{\left(\xi_{\mathbf{k},-}+p_{\lambda} S_{k_{z}}\right)^{2}+\left|S_{\mathbf{k}_{\perp}}\right|^{2}}+p_{\lambda} \xi_{\mathbf{k},+}
$$

are the quasiparticle and quasihole excitation energies. The calculation of the fourth-order fluctuations is lengthy and straightforward, but the results are not particularly illuminating. Next we use the fluctuation action to study the time-dependent Ginzburg-Landau functional near $T_{c}$.

\section{A. Ginzburg-Landau theory near $T_{c}$}

The Ginzburg-Landau theory is used to study the lowfrequency and long-wavelength behavior of the order parameter near $T_{c}$. For this purpose, first we consider the static part of the propagator and expand $L^{-1}(\mathbf{q}, 0)$ in powers of $q_{i}$, and then expand $L^{-1}\left(\mathbf{0}, v_{n}\right)-L^{-1}(\mathbf{0}, 0)$ in powers of $\omega$ after the analytic continuation $i v_{n} \rightarrow \omega+i 0^{+}$.

This calculation leads to the time-dependent Ginzburg-Landau equation in $\mathbf{k}$ space [26,27], i.e.,
$L^{-1}(\mathbf{q}, \omega)=a(T)+\sum_{i, j} c_{i j} q_{i} q_{j} / 2-d \omega$. Here, the zeroorder coefficient $L^{-1}(\mathbf{0}, 0)$ is given by

$$
\begin{aligned}
a(T)= & \frac{1}{g}-\frac{1}{8} \sum_{\mathbf{k}, \lambda_{o}, \lambda_{e}} \frac{X_{\mathbf{k}, \lambda_{o}}-X_{\mathbf{k}, \lambda_{e}}}{E_{\mathbf{k}, \lambda_{o}}-E_{\mathbf{k}, \lambda_{e}}} \\
& \times\left[1-s_{\lambda_{o}} p_{\lambda_{e}} \frac{\xi_{\mathbf{k},-}^{2}-\left|S_{\mathbf{k}_{\perp}}\right|^{2}-S_{k_{z}}^{2}}{\sqrt{\left(C_{\mathbf{k},+}^{2}+\left|S_{\mathbf{k}_{\perp}}\right|^{2}\right)\left(C_{\mathbf{k},-}^{2}+\left|S_{\mathbf{k}_{\perp}}\right|^{2}\right)}}\right] .
\end{aligned}
$$

The condition $a\left(T_{c}\right)=0$ is the Thouless criterion, and it leads to an equation for $T_{c}$. We checked for all four types of SOC that this criterion is in agreement with the order parameter equation after setting $|\Delta|=0$ in the latter. The coefficient of the time-dependent term

$$
\begin{aligned}
d= & \frac{1}{8} \sum_{\mathbf{k}, \lambda_{o}, \lambda_{e}}\left(X_{\mathbf{k}, \lambda_{o}}-X_{\mathbf{k}, \lambda_{e}}\right) \\
& \times\left[1-s_{\lambda_{o}} p_{\lambda_{e}} \frac{\xi_{\mathbf{k},-}^{2}-\left|S_{\mathbf{k}_{\perp}}\right|^{2}-S_{k_{z}}^{2}}{\sqrt{\left(C_{\mathbf{k},+}^{2}+\left|S_{\mathbf{k}_{\perp}}\right|^{2}\right)\left(C_{\mathbf{k},-}^{2}+\left|S_{\mathbf{k}_{\perp}}\right|^{2}\right)}}\right] \\
& \times\left[\frac{1}{\left(E_{\mathbf{k}, \lambda_{o}}-E_{\mathbf{k}, \lambda_{e}}\right)^{2}}+\frac{i \delta\left(E_{\mathbf{k}, \lambda_{o}}-E_{\mathbf{k}, \lambda_{e}}-\omega\right)}{\omega}\right]
\end{aligned}
$$

is a complex number. For balanced Fermi gases, while its imaginary part reflects the decay of Cooper pairs into the twoparticle continuum for $\mu>0$, the imaginary part vanishes for $\mu<0$ and the behavior of the fluctuation field is propagating, reflecting the presence of stable bound states (molecular bosons) [26,27].

The second-order coefficient $\partial^{2} L^{-1}(\mathbf{q}, 0) / \partial q_{i} \partial q_{j}$ evaluated at $\mathbf{q}=0$ is not illuminating for imbalanced Fermi gases. However, for balanced Fermi gases with a PRD-type SOC, where $c_{x x}=c_{y y}=c_{\perp}, c_{z z}=c_{z}$, and $c_{i \neq j}=0$, the coefficients are given by

$$
\begin{aligned}
c_{i j}= & \frac{1}{16 m} \sum_{\mathbf{k}, s}\left\{\frac{X_{\mathbf{k}, s} Y_{\mathbf{k}, s}}{2 m T^{2} E_{\mathbf{k}, \mathbf{s}}} k_{i} k_{j}\left(1+s \frac{m \alpha}{k_{\perp}}\right)^{2}\right. \\
& -\frac{Y_{\mathbf{k}, s}}{2 T E_{\mathbf{k}, \mathbf{s}}}\left[\delta_{i j}\left(1+s \frac{m \alpha}{k_{\perp}}\right)^{2}-s \frac{m \alpha}{k_{\perp}} \frac{k_{i} k_{j}}{k_{\perp}^{2}}\right] \\
& \left.+\frac{X_{\mathbf{k}, s}}{E_{\mathbf{k}, \mathbf{s}}^{2}}\left[\delta_{i j}+s \frac{m \alpha}{k_{\perp}}\left(\delta_{i j}-\frac{k_{i} k_{j}}{k_{\perp}^{2}}\right)\right]\right\} \\
& +\frac{1}{8} \sum_{\mathbf{k}, s, s^{\prime}} \frac{X_{\mathbf{k}, s}+X_{\mathbf{k}, s^{\prime}}}{E_{\mathbf{k}, s}+E_{\mathbf{k}, s^{\prime}}} \frac{s s^{\prime}}{k_{\perp}^{2}}\left(\delta_{i j}-\frac{k_{i} k_{j}}{k_{\perp}^{2}}\right),
\end{aligned}
$$

where $E_{\mathbf{k},+(-)}= \pm E_{\mathbf{k}, 1(2)}$. One needs to set the explicit $\alpha$ terms and the last $\sum_{\mathbf{k}, s, s^{\prime}}$ term to zero to extract $c_{z}$ from this expression. For balanced Fermi gases with a FS-type SOC, where $c_{i i}=c_{0}$ and $c_{i \neq j}=0$, one simply needs to replace $k_{\perp}$ with $k$ to extract $c_{0}$. Similarly, in the case of the ERD-type SOC, where $c_{x x} \neq c_{y y}=c_{z z}$ and $c_{i \neq j}=0$, one needs to replace $k_{\perp}$ with $k_{x}$ to extract $c_{x x}$, and set the explicit $\alpha$ terms and the last $\sum_{\mathbf{k}, s, s^{\prime}}$ term to zero to extract $c_{y y}=c_{z z}$. Finally, in the case of the FA-type SOC, where $c_{x x}=c_{y y} \neq c_{z z}$ and $c_{i \neq j}=0$, one needs to replace $k_{\perp}$ with $k_{z}$ 
to extract $c_{z z}$, and set the explicit $\alpha$ terms and the last $\sum_{\mathbf{k}, s, s^{\prime}}$ term to zero to extract $c_{x x}=c_{y y}$.

In general, the coefficients $a(T), d$, and $c_{i i}$ need to be calculated numerically together with the order parameter and number equations. However, it can be analytically shown that their asymptotic forms recover the usual Ginzburg-Landau equation for BCS superfluids in weak coupling and the GrossPitaevskii equation for a weakly interacting dilute Bose gas in strong coupling [26,27]. Next we use the latter correspondence to extract the effective mass of the Cooper pairs (molecular bosons) and their critical condensation temperature in the molecular BEC limit.

\section{B. Molecular BEC limit}

For illustration purposes, here we consider only balanced Fermi gases. In the molecular BEC limit, when $\mu<0$ and $|\mu| \gg T_{c}$ so that $E_{\mathbf{k}, s} / T \rightarrow \infty$, we may set $X_{\mathbf{k}, s} \rightarrow 1$ and $Y_{\mathbf{k}, s} \rightarrow 0$. Therefore, in this limit, the time-dependent coefficient simplifies to $d=$ $\sum_{\mathbf{k}, s} 1 /\left(8 E_{\mathbf{k}, s}^{2}\right)$ for all types of SOC. The second-order coefficients simplify to $c_{\perp}=\sum_{\mathbf{k}, s}\left[1+s m \alpha /\left(2 k_{\perp}\right)\right] /\left(16 m E_{\mathbf{k}, s}^{2}\right)+$ $\sum_{\mathbf{k}, s, s^{\prime}} s s^{\prime} /\left[8 k_{\perp}^{2}\left(E_{\mathbf{k}, s}+E_{\mathbf{k}, s^{\prime}}\right)\right]$ along the $(x, y)$ directions and to $c_{z}=\sum_{\mathbf{k}, s} 1 /\left(16 m E_{\mathbf{k}, s}^{2}\right)$ along the $z$ direction for the PRD-type SOC, and similarly to $c_{0}=\sum_{\mathbf{k}, s}[1+2 s m \alpha /$ $(3 k)] /\left(16 m E_{\mathbf{k}, s}^{2}\right)+\sum_{\mathbf{k}, s, s^{\prime}} s s^{\prime} /\left[6 k^{2}\left(E_{\mathbf{k}, s}+E_{\mathbf{k}, s^{\prime}}\right)\right]$ along all $(x, y, z)$ directions for the FS-type SOC. In the case of ERDand FA-type SOCs, we note that since $\left(\delta_{i j}-k_{i} k_{j} / k_{x}^{2}\right)=0$ for $i=j=x$ and $\left(\delta_{i j}-k_{i} k_{j} / k_{z}^{2}\right)=0$ for $i=j=z$, respectively, the diagonal coefficients all become equal in the molecular limit, i.e., $c_{0}=c_{i i}$, and it is $c_{0}=\sum_{\mathbf{k}, s} 1 /\left(16 m E_{\mathbf{k}, s}^{2}\right)$.

These k-space sums are analytically tractable for all four types of SOC that we consider in this paper. For instance, for the ERD- or FA-type SOC, we obtain $d=2 m c_{0}=$ $m \sqrt{m} V /\left(8 \pi \sqrt{2|\mu|-m \alpha^{2}}\right)$. However, for the PRD-type SOC, we obtain $d=m \sqrt{2 m|\mu|} V /\left[8 \pi\left(2|\mu|-m \alpha^{2}\right)\right]$ for the time-dependent coefficients, and $c_{\perp}=\sqrt{2 m} V\left(4|\mu|-m \alpha^{2}\right) /$ $\left[64 \pi \sqrt{|\mu|}\left(2|\mu|-m \alpha^{2}\right)\right]-\sqrt{2 m} /[64 \pi \sqrt{|\mu|}] \ln \left[\left(2|\mu|-m \alpha^{2}\right) /\right.$ $(2|\mu|)]$ along the $(x, y)$ directions and $c_{z}=$ $m^{2} \sqrt{2 m|\mu|} V /\left[4 \pi\left(2|\mu|-m \alpha^{2}\right)\right]$ along the $z$ direction for the second-order coefficients. Similarly, for the FS-type SOC, we obtain $d=m \sqrt{m}|\mu| V /\left[4 \pi\left(2|\mu|-m \alpha^{2}\right)^{3 / 2}\right]$ for the time-dependent coefficients and $c_{0}=\sqrt{m}(7|\mu|-$ $\left.3 m \alpha^{2}\right) V /\left[24 \pi\left(2|\mu|-m \alpha^{2}\right)^{3 / 2}\right]-\sqrt{m} /(12 \pi \sqrt{2|\mu|})$ for the second-order coefficients. Next we extract the effective Gross-Pitaevskii parameters using the asymptotic forms of the Ginzburg-Landau coefficients given above.

\section{Effective molecular mass}

We recall that, since the Ginzburg-Landau theory derived above reduces to the Gross-Pitaevskii theory of a weakly interacting molecular Bose gas after the rescaling $\Psi(\mathbf{q}, \omega)=$ $\sqrt{d} \Lambda(\mathbf{q}, \omega)$, the effective mass of the Cooper pairs (molecular bosons) along the $i$ th direction is simply given by $m_{B, i}=d / c_{i i}$ $[26,27]$. In the absence of a SOC, this gives $m_{B}=2 m$ for all $(x, y, z)$ directions.

Using the asymptotic forms of $d$ and $c_{0}$, and setting $|\mu|=\left(\left|\epsilon_{b}\right|+m \alpha^{2}\right) / 2$, we find that the mass of the molecular bosons is $m_{B, x}=m_{B, y}=m_{B, z}=m_{B}=2 m$ for the ERD- and

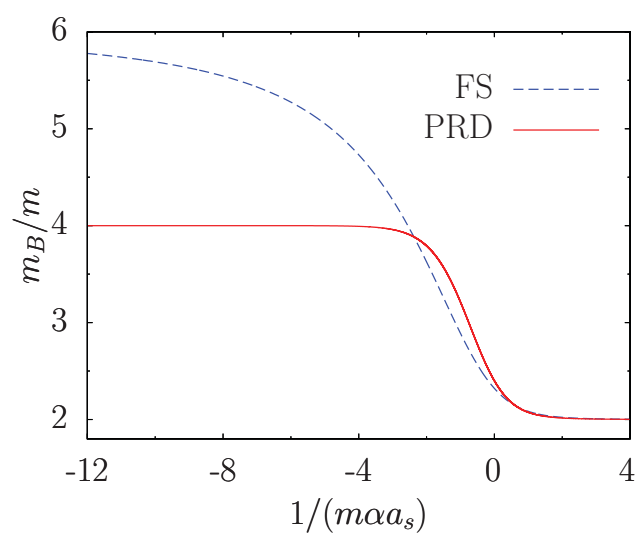

FIG. 6. (Color online) The effective mass of the Cooper pairs are shown in the molecular BEC limit as a function $1 /\left(m \alpha a_{s}\right)$ for the PRD- and FS-type SOCs.

FA-type SOCs. However, using the asymptotic forms of $d, c_{\perp}$, and $c_{z}$, and setting $|\mu|=\left(\left|\epsilon_{b}\right|+m \alpha^{2}\right) / 2$, we find that the mass of the molecular bosons $m_{B, x}=m_{B, y}=m_{B, \perp}$ is

$\frac{2 m}{m_{B, \perp}}=\frac{2\left|\epsilon_{b}\right|+m \alpha^{2}}{2\left|\epsilon_{b}\right|+2 m \alpha^{2}}-\frac{\left|\epsilon_{b}\right|}{2\left|\epsilon_{b}\right|+2 m \alpha^{2}} \ln \left(\frac{\left|\epsilon_{b}\right|}{\left|\epsilon_{b}\right|+m \alpha^{2}}\right)$,

and $m_{B, z}=2 m$ along the $z$ direction for the PRD-type SOC. This expression is in complete agreement with the recent findings [14,15], showing that $m_{B, \perp}$ decreases monotonically with increasing $1 /\left(m \alpha a_{s}\right)$ as plotted in Fig. 6. Equation (18) gives $m_{B}=4 m$ when $1 /\left(m \alpha a_{s}\right) \rightarrow-\infty, m_{B}=2 m$ when $1 /\left(m \alpha a_{s}\right) \rightarrow \infty$, and $m_{B} \approx 2.40 m$ at unitarity when $1 /\left(m \alpha a_{s}\right) \rightarrow 0$. On the other hand, we find that the mass of the molecular bosons $m_{B, x}=m_{B, y}=m_{B, z}=m_{B}$ is

$$
\frac{2 m}{m_{B}}=\frac{7}{3}-\frac{4}{3}\left(\frac{\left|\epsilon_{b}\right|}{\left|\epsilon_{b}\right|+m \alpha^{2}}\right)^{3 / 2}-\frac{2 m \alpha^{2}}{\left|\epsilon_{b}\right|+m \alpha^{2}}
$$

for the FS-type SOC, which is also a monotonically decreasing function of $1 /\left(m \alpha a_{s}\right)$, as shown in Fig. 6. Equation (19) gives $m_{B}=6 m$ when $1 /\left(m \alpha a_{s}\right) \rightarrow-\infty, m_{B}=2 m$ when $1 /\left(m \alpha a_{s}\right) \rightarrow \infty$, and $m_{B}=3 \sqrt{2} m /(2 \sqrt{2}-1) \approx 2.32 m$ at unitarity. Having calculated the effective mass of the Cooper pairs, we are ready to calculate their critical BEC temperature.

\section{Critical BEC temperature}

The $\alpha$ dependence of the Cooper-pair mass in the molecular BEC limit has a dramatic effect on the finite $T$ phase diagram of the system. For atomic Bose gases, $T_{\mathrm{BEC}}$ is determined from the number equation $N_{B}=\sum_{\mathbf{k}} 1 /\left(e^{\epsilon_{\mathbf{k}, B} / T_{\mathrm{BEC}}}-1\right)$, where $N_{B}$ is the number and $\epsilon_{\mathbf{k}, B}=\sum_{i=\{x, y, z\}} k_{i}^{2} /\left(2 m_{B, i}\right)$ is the kinetic energy of atomic bosons, with $m_{B, i}$ their effective mass along the $i$ th direction. This leads to $T_{\mathrm{BEC}}=2 \pi\left\{n_{B} /\left[\sqrt{\Pi_{i} m_{B, i}} \zeta(3 / 2)\right]\right\}^{2 / 3}$ in three dimensions, where $n_{B}=N_{B} / V$ is the density of bosons and $\zeta(x)$ is the Riemann zeta function with $\zeta(3 / 2) \approx$ 2.61. Setting $n_{B}=n / 2$, where $n=N / V=k_{F}^{3} /\left(3 \pi^{2}\right)$ is the total density of fermions, we obtain

$$
T_{\mathrm{BEC}} \approx 0.218 \frac{2 m}{\left(\prod_{i=\{x, y, z\}} m_{B, i}\right)^{1 / 3}} \epsilon_{F}
$$




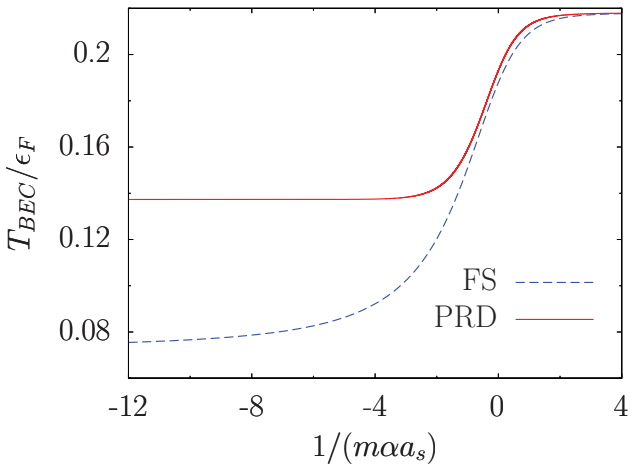

FIG. 7. (Color online) The critical BEC temperature of the Cooper pairs (molecular bosons) are shown as a function $1 /\left(m \alpha a_{s}\right)$ for the PRD- and FS-type SOCs.

in three dimensions, where $\epsilon_{F}=k_{F}^{2} /(2 m)$ is the Fermi energy and $m_{B, i}$ is the mass of the molecular bosons along the $i$ th direction. In the absence of a SOC, this gives $T_{\mathrm{BEC}} \approx$ $0.218 \epsilon_{F}[26]$.

Since we expect the Cooper pairs to progressively become weakly repulsive in the molecular BEC limit, and using the effective mass of the Cooper pairs found in Sec. IV B 1 , we find $T_{\mathrm{BEC}} \approx 0.218 \epsilon_{F}$ for the ERD- and FA-type SOCs, $T_{\mathrm{BEC}} \approx 0.218\left(2 \mathrm{~m} / m_{B, \perp}\right)^{2 / 3} \epsilon_{F}$ for the PRD-type SOC, and $T_{\mathrm{BEC}} \approx 0.218\left(2 \mathrm{~m} / m_{B}\right) \epsilon_{F}$ for the FS-type SOC. In Fig. 7, we show $T_{\mathrm{BEC}}$ as a function $1 /\left(m \alpha a_{s}\right)$. While $T_{\mathrm{BEC}}$ is independent of $\alpha$ for the ERD- and FA-type SOCs, we find that $T_{\mathrm{BEC}}$ increases monotonically with increasing $1 /\left(m \alpha a_{s}\right)$ for the PRD- and FS-type SOCs. For the PRD-type SOC, we find $T_{\mathrm{BEC}} \approx 0.137 \epsilon_{F}$ when $1 /\left(m \alpha a_{s}\right) \rightarrow-\infty, T_{\mathrm{BEC}} \approx 0.218 \epsilon_{F}$ when $1 /\left(m \alpha a_{s}\right) \rightarrow \infty$, and $T_{\mathrm{BEC}} \approx 0.193 \epsilon_{F}$ at unitarity $[14,15]$. Similarly, for the FS-type SOC, we find $T_{\mathrm{BEC}} \approx$ $0.0726 \epsilon_{F}$ when $1 /\left(m \alpha a_{s}\right) \rightarrow-\infty, T_{\mathrm{BEC}} \approx 0.218 \epsilon_{F}$ when $1 /\left(m \alpha a_{s}\right) \rightarrow \infty$, and $T_{\mathrm{BEC}} \approx 0.188 \epsilon_{F}$ at unitarity.

\section{CONCLUSIONS}

To conclude, we extended our recent works [19,21], and investigated the effects of an anisotropic SOC on the phase diagrams of both balanced and imbalanced Fermi gases throughout the entire BCS-BEC evolution. We analyzed both zero- and finite-temperature phase diagrams, and our main results can be summarized as follows.

In the first part, we derived the self-consistent meanfield theory at zero temperature, and used it to investigate the effects of SOC on the ground-state phase diagrams.
We showed that while both the ERD- and FA-type SOCs do not have any observable effect on the balanced Fermi gases, only the FA-type SOC does not have any effect on the population-imbalanced gases. On the other hand, in the case of ERD- and PRD-type SOCs, we found that the competition between the population imbalance and the SOC gives rise to very rich phase diagrams, involving normal, superfluid, and phase-separated regions, and quantum phase transitions between the topologically trivial gapped superfluid and the nontrivial gapless superfluid phases. For instance, one of the intriguing effects of the SOC is that, in sharp contrast to the no-SOC case where only the gapless superfluid phase supports population imbalance, both the gapless and gapped superfluid phases can support population imbalance in the presence of a SOC. We also showed that the topological structure of the ground-state phase diagrams is quite robust against the effects of anisotropy, i.e., they are very similar for ERD- and PRD-type SOCs.

In the second part, we went beyond the mean-field description, and investigated the effects of Gaussian fluctuations near the critical temperature. This allowed us to derive the timedependent Ginzburg-Landau theory, from which we extracted the effective mass of the Cooper pairs and their critical condensation temperature $T_{\mathrm{BEC}}$ in the molecular BEC limit. We showed that while the effective mass $\left(T_{\mathrm{BEC}}\right)$ of the bosons does not depend on $\alpha$ for the ERD- and FA-type SOCs, it decreases (increases) monotonically as a function of increasing $1 /\left(m \alpha a_{s}\right)$ for the PRD- and FS-type SOCs. We found $T_{\mathrm{BEC}} \approx$ $0.14 \epsilon_{F}$ for the PRD-type SOC and $T_{\mathrm{BEC}} \approx 0.073 \epsilon_{F}$ for the FS-type SOC in the weakly interacting $a_{s} \rightarrow 0^{-}$limit, and $T_{\mathrm{BEC}} \approx 0.19 \epsilon_{F}$ for both types at unitarity when $\left|a_{s}\right| \rightarrow \infty$. This shows that the presence of either a PRD- or FS-type SOC increases $T_{c}$ considerably especially in the BCS limit, which is in sharp contrast to ERD- or FA-type SOCs where the SOC does not have any effect on $T_{c}$ throughout the BCS-BEC evolution.

\section{ACKNOWLEDGMENTS}

This work is supported by the Marie Curie International Reintegration (Grant No. FP7-PEOPLE-IRG-2010-268239), Scientific and Technological Research Council of Turkey (Career Grant No. TÜBITAK-3501-110T839), and the Turkish Academy of Sciences (TÜBA-GEBIP). Computing resources used in this work were provided by the Istanbul Technical University, Informatics Institute, High Performance Computing Laboratory (HPCL Grant No. 1005201003).
[1] M. Inguscio, W. Ketterle, and C. Salomon, Ultra-cold Fermi Gases, Proceedings of the International School of Physics Enrico Fermi, Course CLXIV, Varenna (IOS, Amsterdam, 2008).

[2] S. Giorgini, L. P. Pitaevskii, and S. Stringari, Rev. Mod. Phys. 80, 1215 (2008).

[3] M. W. Zwierlein, A. Schirotzek, C. H. Schunck, and W. Ketterle, Science 311, 492 (2006).

[4] G. B. Partridge, W. Li, R. I. Kamar, Y. Liao, and R. G. Hulet, Science 311, 503 (2006).
[5] Y. Shin, C. H. Schunck, A. Schirotzek, and W. Ketterle, Nature (London) 451, 689 (2008).

[6] N. Navon, S. Nascimbéne, F. Chevy, and C. Salomon, Science 328, 729 (2010).

[7] M. Sato, Y. Takahashi, and S. Fujimoto, Phys. Rev. Lett. 103, 020401 (2009).

[8] A. Kubasiak, P. Massignan, and M. Lewenstein, Europhys. Lett. 92, 46004 (2010).

[9] Y.-J. Lin, R. L. Compton, A. R. Perry, W. D. Phillips, J. V. Porto, and I. B. Spielman, Phys. Rev. Lett. 102, 130401 (2009). 
[10] Y.-J. Lin, Y.-J. Lin, K. Jiménez-García, and I. B. Spielman, Nature (London) 471, 83 (2011).

[11] J. D. Sau, R. Sensarma, S. Powell, I. B. Spielman, and S. Das Sarma, Phys. Rev. B 83, 140510 (2011).

[12] J. P. Vyasanakere and V. B. Shenoy, Phys. Rev. B 83, 094515 (2011).

[13] J. P. Vyasanakere, S. Zhang, and V. B. Shenoy, Phys. Rev. B 84, 014512 (2011)

[14] Z.-Q. Yu and H. Zhai, e-print arXiv:1105.2250.

[15] Hui Hu, L. Jiang, X.-J. Liu, and Han Pu, e-print arXiv:1105.2488.

[16] Li Han and C. A. R. Sá de Melo, e-print arXiv:1106.3613.

[17] G. Chen, M. Gong, and C. Zhang, e-print arXiv:1107.2627.

[18] M. Gong, S. Tewari, and C. Zhang, e-print arXiv:1105.1796.
[19] M. Iskin and A. L. Subaşi, Phys. Rev. Lett. 107, 050402 (2011). [20] W. Yi and G.-C. Guo, e-print arXiv:1106.5667.

[21] M. Iskin and A. L. Subaşi, e-print arXiv:1107.2376.

[22] L. P. Gor'kov and E. I. Rashba, Phys. Rev. Lett. 87, 037004 (2001).

[23] G. Dresselhaus, Phys. Rev. 100, 580 (1955).

[24] Note that the FA-type SOC may not be eliminated by a momentum shift for mass-imbalanced Fermi gases.

[25] J. P. Gaebler, J. T. Stewart, T. E. Drake, D. S. Jin, A. Perali, P. Pieri, and G. C. Strinati, Nat. Phys. 6, 569 (2010).

[26] C. A. R. Sá de Melo, M. Randeria, and J. R. Engelbrecht, Phys. Rev. Lett. 71, 3202 (1993).

[27] M. Iskin and C. A. R. Sá de Melo, Phys. Rev. A 74, 013608 (2006). 